\title{
La protection des indications géographiques : les enjeux du mandat de Doha
}

\section{Mai-Anh Ngo}

\section{(2) OpenEdition}

1 Journals

\section{Édition électronique}

URL : http://journals.openedition.org/economierurale/1913

DOI : 10.4000/economierurale.1913

ISSN : 2105-2581

\section{Éditeur}

Société Française d'Économie Rurale (SFER)

\section{Édition imprimée}

Date de publication : 1 novembre 2006

Pagination : 117-123

ISSN : 0013-0559

\section{Référence électronique}

Mai-Anh Ngo, "La protection des indications géographiques : les enjeux du mandat de Doha »,

Économie rurale [En ligne], 294-295 | Juillet-octobre 2006, mis en ligne le 29 octobre 2009, consulté le 30 avril 2019. URL : http://journals.openedition.org/economierurale/1913 ; DOI : 10.4000/

economierurale.1913 


\title{
La protection des indications géographiques: les enjeux du mandat de Doha
}

Mai-Anh NGO • CNRS, Groupe de recherche en droit, économie et gestion, Centre de recherche en droit économique, GREDECO-GREDEG

\begin{abstract}
Depuis une dizaine d'années, d'âpres discussions sont menées au sein de l'Organisation mondiale du commerce concernant l'Accord sur les droits de propriété intellectuelle qui touchent au commerce et, plus particulièrement, les indications géographiques. De nombreux pays sont en désaccord sur ce sujet car les enjeux commerciaux sont importants. Des améliorations sont envisagées. L'auteur de ce débat en fait le point. Il nous a semblé intéressant de porter à la connaissance de nos lecteurs la manière dont a été traitée cette question qui va conférer au produit alimentaire une dimension mondiale (NDLR).
\end{abstract}

a protection des indications géographiques -dans le cadre de l'OMC ${ }^{1}$, par le biais des accords $\mathrm{ADPIC}^{2}$, présente un intérêt particulier dans la mesure où, d'une part, les indications géographiques sont expressément prévues comme des éléments de propriété intellectuelle et, d'autre part, en vertu de la règle de l'engagement unique de l'accord ADPIC, les engagements qu'il contient, notamment en matière d'indications géographiques, deviennent contraignants pour l'ensemble des membres de l'OMC.

La section 3 de l'accord ADPIC institue une réglementation propre aux indications géographiques qui assure une protection du consommateur et du producteur, répondant ainsi à la fois aux objectifs concurrentiels et consuméristes. De sorte, elle évite au consommateur d'être induit en erreur sur l'origine géographique du produit et sur les qualités qui en découlent. Elle garantit également au producteur le bénéfice qu'emporte l'indication géographique répondant véritablement aux critères définis.

1. Organisation mondiale du commerce.

2. Aspects des droits de propriété industrielle qui touchent au commerce.
L'accord ADPIC établit un double niveau de protection : le premier niveau qui s'applique à tous les produits, à l'exception des vins et spiritueux, peut être considéré comme étant de portée générale, le second, au contraire, est réservé aux seuls vins et spiritueux et offre des garanties supérieures.

L'étendue de la protection conférée par l'accord ADPIC reste, de manière générale, relativement limitée, ceci s'explique sans doute par la volonté d'obtenir l'adhésion du plus grand nombre possible d'États. La conférence ministérielle de l'OMC à Doha, en 2001, a réaffirmé la nécessité de compléter la partie de l'accord ADPIC consacrée aux indications géographiques conformément aux articles $23 \S 4$ et $24 \S 1$ de l'accord ADPIC.

Les évolutions envisagées font l'objet d'âpres débats au sein du conseil des ADPIC, les oppositions entre les différents pays sont extrêmement marquées car les enjeux liés à ces questions sont énormes. Dans ce débat ${ }^{3}$, la première partie discutera

3. Une première version de ce texte a déjà été présentée lors du colloque de la Sfer sur «Au nom de la qualité : quelles qualité(s) demain, pour quelle(s) demande $(s)$ ? », Clermont-Ferrand, 5-6 octobre 2005. 
de la première amélioration consistant à créer un système multilatéral de notification et d'enregistrement des indications géographiques pour les vins. La seconde amélioration, analysée dans la seconde partie, vise à étendre le niveau de protection des vins et spiritueux, plus élevé, aux autres produits.

\section{L'établissement d'un registre multilatéral d'indications géographiques pour les vins}

Le président du conseil des ADPIC affirme que l'instauration d'un registre multilatéral ${ }^{4}$ pour les indications géographiques en matière de vins n'a pas pour objectif et ne doit pas influer sur l'étendue de la protection conférée à ce type de produit ${ }^{5}$. Ceci s'explique par la volonté de ne pas heurter les pays peu favorables à une réglementation rigoureuse en matière d'indications géographiques.

Cependant la réalité est tout autre. Selon, le type de registre multilatéral choisi, les effets juridiques seront plus ou moins importants. Le choix d'un système de notification ne fait que faciliter le respect des obligations actuelles, alors que le fait d'opter pour l'établissement d'un système d'enregistrement accroît véritablement le niveau de protection.

\section{Un système de notification facilitant le respect des obligations actuelles}

La création d'un système volontaire de notification des indications géographiques dans une base de données est soutenue par nombreux pays, dont le Canada et les

\footnotetext{
4. Par souci de concision, nous parlons d'un registre multilatéral d'indications géographiques, mais selon les termes de la déclaration de Doha $§ 18$ il s'agit d'un système multilatéral de notification et d'enregistrement des indications géographiques.

5. OMC, Compte rendu de la réunion tenue le 28 juin 2002, document TN/IP/M/2 § 141, http://www.wto.org.
}

États-Unis ${ }^{6}$. Les gouvernements ayant choisi de participer au système devraient notifier leurs indications géographiques et s'engager à consulter la base de données lorsqu'ils qualifieraient une indication afin de lui accorder ou non une protection. Cette décision demeure toutefois prise en fonction de la législation nationale, le registre ne constitue qu'une source d'information. De plus les membres n'ayant pas adhéré au système ne sont pas tenus de le faire. En l'occurrence, il s'agirait uniquement d'une compilation des différentes notifications, ayant un but informatif. Cette proposition présente cependant l'avantage d'améliorer la transparence du système actuel. Les instances nationales chargées de prendre les décisions quant à la protection ou non d'une dénomination disposeraient ainsi d'une vision exhaustive concernant l'éventuelle protection de la dénomination litigieuse. En effet actuellement, en vertu de l'accord ADPIC, il n'existe aucune obligation de protection préalable dans le pays d'origine avant de solliciter une protection internationale. De plus, à l'heure actuelle, les offices nationaux de propriété intellectuelle ne disposent d'aucune base de données permettant de connaître la qualification d'une indication géographique.

Une telle solution ne crée substantiellement aucune nouvelle obligation à la fois pour les pays participants et pour les pays non participants. Sa mise en œuvre n'a pas réellement d'incidence juridique et semble relativement peu coûteuse. Ce choix n'est pas surprenant dans la mesure où les rédacteurs du projet sont pour partie américains. En effet, aux États-Unis la liberté du commerce est érigée au rang de libertés fondamentales. Suivant cette logique, toute réglementation destinée à protéger les indications de provenance est a priori perçue

6. OMC, Proposition relative un système multilatéral de notification et d'enregistrement des indications géographiques pour les vins et spiritueux, document TN/IP/W/5. 
comme une entrave. De plus, il faut souligner que les États-Unis accordent peu d'importance aux indications géographiques car les marques occupent une place prépondérante dans ce pays. Le fait de ne retenir qu'un système de notification est une position conforme à leur réglementation.

Ce choix ne présente cependant pas que des avantages, en particulier en raison de l'incertitude juridique importante que ce système engendre ${ }^{7}$. En effet, ce n'est qu'à l'occasion d'un litige qu'il sera possible de déterminer si la dénomination est susceptible d'être protégée. Concrètement, cela permet à chaque pays de refuser unilatéralement la protection à une indication géographique. Ceci est particulièrement dangereux aux États-Unis qui ont une conception très élargie de la dégénérescence des dénominations ${ }^{8}$. D'après le droit américain, le fait qu'une indication géographique soit considérée comme générique ou semi-générique exclut toute possibilité de protection pour le terme en question. Le semi-générique étant " un nom ayant une signification géographique qui est également la désignation d'une catégorie ou d'un type de vins. Il doit être modifié par la description du véritable lieu d'origine du vin »'. Un terme est, quant à lui, considéré comme générique à partir du moment où «le nom d'un produit agricole ou d'une denrée alimentaire qui, bien que se rapportant au lieu ou à la région où ces produits agricoles ou cette denrée alimentaire a été initialement produit ou commercialisé, est devenu le nom commun d'un produit agricole ou d'une denrée alimentaire ${ }^{10}$. Cela conduit, en fait, à refuser toute protec-

7. Rochard D. (2002). La protection internationale des indications géographiques. Paris, PUF, p. 22-42. 8. L'Union européenne a cependant réussi à requalifier certains génériques grâce à l'accord signé avec les États-Unis, sur le commerce des vins, le 15 septembre 2005.

9. Cf. Schaeffer E. (2000). Les signes de qualité et d'origine. Revue des chambres de l'agriculture, $\mathrm{n}^{\circ} 884, \mathrm{p} 27$.

10. Article 3 du règlement 510/2006. tion dès l'instant où le consommateur ne fait pas le lien entre la qualité du produit et son origine. En plus de la divergence spatiale, une hétérogénéité temporelle peut voir le jour. La dégénérescence a, par nature, un caractère évolutif. Il arrive en effet que certains produits soient à tel point imités que le nom qu'ils portent finit par ne plus désigner que le type du produit qu'il représente.

La présentation succincte de ce système de notification démontre qu'il ne s'agit en aucun cas de la solution idéale. C'est la raison pour laquelle d'autres propositions ont été faites par d'autres pays membres de l'OMC. Ces différents projets suggèrent l'instauration d'un système d'enregistrement accroissant le niveau de protection des indications géographiques.

\section{Un système d'enregistrement accroissant le niveau de protection}

Les États favorables à l'établissement d'un système multilatéral d'enregistrement plus rigoureux sont à l'origine de différentes propositions. En réalité, les textes européens ${ }^{11}$ et chinois ${ }^{12}$ envisagent l'instauration d'un système d'enregistrement conférant une présomption de protection dans tous les pays et pour toutes les indications géographiques enregistrées. La Chine et Hong Kong prévoient toutefois qu'un État pourra refuser la protection à une indication géographique conformément à sa législation nationale ${ }^{13}$. De plus, seuls les États ayant choisi de participer au système d'enregistrement seront contraints par celui-ci.

11. OMC, Proposition relative un système multilatéral de notification et d'enregistrement des indications géographiques pour les vins et spiritueux, document IP/C/W/107/Rev.1, 22 juin 2000, document TN/IP/W/10, 14 juin 2005.

12. OMC, Proposition relative un système multilatéral de notification et d'enregistrement des indications géographiques pour les vins et spiritueux, document TN/IP/W/8, 23 avril 2003.

13. OMNC, Proposition relative un système multilatéral de notification et d'enregistrement des indications géographiques pour les vins et spiritueux, document TN/IP/W/8, ibid. 
Dans tous ces projets, il est possible, contrairement à la proposition américaine, de distinguer la notification de l'enregistrement. Ceci permet d'assurer au moins à chaque fois un examen formel de la demande. De sorte, il est possible de s'assurer qu'aucune protection ne soit indûment conférée à une indication géographique. Au sein de l'Union européenne, la valorisation de l'origine occupe une place très importante au sein de diverses politiques communautaires, telles que la politique agricole commune ou les politiques visant la protection du consommateur. À ce titre il est indispensable que la qualité légale, symbolisée pour partie par l'indication géographique, corresponde à la qualité réelle du produit. Cette volonté d'encadrement rigoureux se retrouve dans la proposition communautaire qui se déroule en quatre phases : la notification, l'examen formel, l'opposition et l'enregistrement.

L'existence d'une présomption de protection pour les indications géographiques enregistrées garantit une meilleure protection dans la mesure où elle oblige les parties souhaitant utiliser la dénomination à réfuter la présomption. Dans les faits, les utilisateurs légitimes de l'indication géographique pourront assurer plus aisément leur défense sur un marché mondialisé.

L'inconvénient de ces diverses propositions réside dans leur coût financier et humain. Il existe en effet une relation entre la complexité du système et les coûts qu'il engendre. Toutefois, les Communautés européennes conscientes du problème ont proposé, lors de leur dernier projet, un système de taxes pour financer le système ${ }^{14}$.

Après avoir réalisé un rapide bilan de l'instauration d'une présomption réfragable, il est nécessaire de revenir plus en détail sur le projet européen, qui prévoit un système d'opposition très précis. Ce texte

14. OMC, Indications géographiques, communication des Communautés européennes, document TN/IP/W/11, 14 juin 2006. instaure un délai de dix-huit mois pendant lequel les différents pays peuvent s'opposer à l'enregistrement. Ceci représente une avancée majeure, notamment pour lutter contre la dégénérescence et pour assurer une plus grande sécurité juridique. Concrètement, la protection de l'indication géographique n'est plus uniquement liée à l'attitude du pays dans lequel la protection est sollicitée. La procédure d'opposition permet de respecter le droit des tiers qui peuvent faire valoir l'éventuel caractère générique d'une dénomination.

Cette formalité supplémentaire constitue, pour les défenseurs d'un registre multilatéral moins contraignant, de nouvelles obligations pesant sur les membres participants ou non. Tous les pays seraient obligés d'examiner l'ensemble des demandes d'enregistrement dans le temps imparti. À la suite de cette procédure d'opposition, une solution peut être trouvée grâce à des négociations bilatérales. Si aucun accord n'est trouvé soit l'État ayant émis sa réserve la retire et l'indication est enregistrée, soit l'État maintient sa réserve, l'indication est enregistrée avec une précision expresse concernant la contestation relative à l'enregistrement.

Malgré certains inconvénients, l'analyse d'un système d'enregistrement semble le plus adapté pour assurer une protection efficace des indications géographiques pour les vins. Même si cela augmente quelque peu le contenu substantiel du texte actuel, cette modification est moins profonde que celle qui concerne l'extension de la protection additionnelle aux autres produits.

\section{L'extension de la protection additionnelle aux autres produits}

En ce qui concerne l'élargissement de la protection additionnelle aux autres produits, l'opposition entre les membres de l'OMC est encore plus forte que pour la question de l'établissement d'un registre multilatéral. Une discussion de fond n'a même pas pu débuter sur le sujet dans la mesure où les 
Etats n'ont pas la même interprétation du $\$ 12$ de la déclaration de Doha. En effet, certains pays considèrent que cette question n'entre pas dans le cadre du mandat de Doha et ne veulent pas entamer des négociations, alors que d'autres estiment le contraire et souhaitent discuter. Pour comprendre l'intensité des débats et mesurer les enjeux d'éventuelles propositions, il est important d'analyser successivement le contenu de la protection générale et celui de la protection additionnelle.

\section{La protection générale : des garanties minimales}

Le régime qui assure la protection des indications géographiques pour d'autres produits que les vins et spiritueux comporte deux aspects : d'un côté, des principes généraux qui concernent l'ensemble des droits de propriété intellectuelle et de l'autre des dispositions spécifiques aux indications géographiques.

Les principes fondamentaux que sont le traitement national et la nation la plus favorisée, sont repris dans les articles 3 et 4 de l'accord ADPIC. L'intérêt de ces dispositions en matière d'indications géographiques est limité ${ }^{15}$. L'étude détaillée des dispositions spécifiques permet de saisir l'étendue des droits dont bénéficient les titulaires des indications géographiques.

Aux termes des $\S 2$ et $\S 3$ de l'article 22 de l'accord ADPIC, les membres doivent protéger les indications géographiques contre l'utilisation de tout moyen qui induit le public en erreur quant à l'origine géographique du produit et contre toute utilisation qui constitue un acte de concurrence déloyale au sens de l'article 10bis de la Convention de l'Union de Paris ${ }^{16}$.

15. $C f$. Ngo M.-A. (2006). La qualité et la sécurité des produits agroalimentaires. Approche juridique. Paris, L'Harmattan.

16. L'article 10bis de la Convention de l'Union de Paris définit la concurrence déloyale comme «tout acte de concurrence contraire aux usages honnêtes ».
La rédaction de ces deux paragraphes implique que la protection des indications géographiques pour les produits autres que les vins et spiritueux est subordonnée à la constatation d'une indication qui trompe ou d'un acte de concurrence déloyale. Ces éléments constitutifs de l'infraction révèlent que l'indication géographique n'est pas protégée en tant que droit privatif.

En plus de la prohibition d'actes de concurrence déloyale, l'accord ADPIC prend soin de gérer les relations entre les autres signes et les indications géographiques.

- L'article $22 \S 3$ pose le principe d'un refus ou d'une invalidation d'une marque de fabrique ou de commerce qui contient une indication géographique ou qui est constituée par une telle indication pour des produits qui ne sont pas originaires du territoire indiqué, si l'utilisation de cette indication dans la marque de fabrique ou de commerce pour de tels produits dans ce pays membre est de nature à induire le public en erreur quant au véritable lieu d'origine.

- Cependant, cette protection est limitée par l'article $24 \S 5$ qui autorise le maintien d'une marque de fabrique de commerce identique ou similaire à une indication géographique à partir du moment où elle $\mathrm{a}$ été enregistrée ou acquise de bonne foi avant l'entrée en vigueur de l'accord, ou avant que l'indication géographique ne soit protégée dans son pays d'origine.

Excepté les rapports entre les indications géographiques et les marques, l'accord interdit les homonymies trompeuses. En somme le contenu de la protection générale conférée est relativement restreint, et il n'est pas surprenant, dès lors que certains États cherchent à favoriser l'extension aux autres produits de la protection additionnelle.

\section{L'extension de la protection additionnelle aux autres produits : vers la reconnaissance d'un droit privatif ?}

La différence fondamentale entre la protection additionnelle et la protection générale réside dans le fait qu'il n'est plus obligatoire 
que le public soit induit en erreur pour que la dénomination soit protégée. De même, il n'est pas nécessaire de s'attacher à la conduite du défendeur pour obtenir une condamnation $^{17}$. La protection des vins et spiritueux est, par conséquent, objective et automatique. Le demandeur doit uniquement apporter la preuve de l'existence et du caractère opposable de son droit ainsi que l'empiétement par le défendeur de son domaine réservé.

Il faut souligner que la portée d'un élargissement aux autres produits va, cependant, bien au-delà de la simple amélioration de la protection. Une telle évolution implique en effet un véritable changement dans la conception même de la protection des indications géographiques reconnues alors comme de véritables droits privatifs ${ }^{18}$.

L'interprétation de l'article $23 \$ 1$ permet aussi de condamner l'utilisation des traductions, des correctifs ${ }^{19}$ et des délocalisants ${ }^{20}$.

L'article $23 \$ 2$ interdit l'enregistrement des indications géographiques comme marques pour les vins et spiritueux non originaire de ce lieu. La protection additionnelle est renforcée par rapport à la protection générale dans la mesure où il n'est plus nécessaire que ces marques soient de nature à induire le public en erreur.

À côté de ces différents prohibitions, l'article $24 \$ 4$ de l'accord ADPIC autorise l'utilisation d'une indication géographique pour les vins et spiritueux lorsque le bénéficiaire

17. Article $23 \S 1$ de l'accord ADPIC.

18. Roubier P. (1954). Le droit de la propriété industrielle. Paris, Sirey.

19. Le terme correctif regroupe l'ensemble des expressions telles que « genre», «type », « style » visant à informer le consommateur du fait qu'il s'agit uniquement d'un produit qui est fabriqué à la manière de l'original, mais qui n'est pas le produit authentique.

20. Le délocalisant est l'indication de l'origine d'un produit, que l'on fait parfois figurer sur les produits qui, pour une raison ou pour une autre, sont susceptibles d'évoquer une provenance différente de leur provenance réelle. domicilié dans un pays membre l'a utilisé au moins pendant dix ans de bonne foi avant le 15 avril 1994. L'exception concernant la coexistence des marques et des indications géographiques est donc reformulée par rapport à la protection générale.

Si la protection pour les autres produits interdit les homonymies qui induisent le public en erreur, en revanche l'article 23 $\S 3$ autorise cette coexistence pour les vins.

$\mathrm{Au}$ terme de cette rapide analyse, il est évident que l'extension de la protection additionnelle aux autres produits constituerait un avantage pour les pays les plus attachés aux indications géographiques. Pour les États plus favorables à une politique de marque, comme les États-Unis, une telle rigueur n'est pas nécessaire ${ }^{21}$. Au-delà d'un accroissement du niveau de protection, cette évolution engendrerait un véritable changement de nature de l'accord ADPIC en matière d'indications géographiques.

En définitive, l'étude des différentes évolutions envisageables pour la protection des indications géographiques par le biais de l'accord ADPIC souligne les divergences profondes qui existent à l'échelle internationale en ce qui concerne les politiques de valorisation de l'origine. Cette analyse se vérifie sur le terrain dans la mesure où les négociations n'ont pas véritablement avancé au sujet des indications géographiques. La suspension du cycle de Doha pourra peut-être constituer une période propice à la réflexion pour permettre l'avancée des négociations.

Il faut insister sur le fait que la mise en place d'un système d'enregistrement pour les vins et l'extension de la protection additionnelle aux autres produits représenteraient sans doute à l'avenir la possibilité de mettre en œuvre une véritable protection

21. $C f$. Lackert C.-W. Indications géographiques : quelles obligations en vertu de l'accord ADPIC ? In la revue Propriété industrielle Bulletin d'information, $P I B D$, de l'Institut national de la propriété industrielle, II, $\mathrm{n}^{\circ} 665$, p. 201. 
universelle des indications géographiques. Dans sa forme la plus aboutie la protection des indications géographiques devrait permettre qu' aucun autre produit au monde ne puisse être désigné grâce à la dénomination protégée, conférant de la sorte à l'échelle planétaire une très forte valeur concurrentielle au produit.
L'auteur tient à remercier les évaluateurs anonymes pour leurs critiques et leurs suggestions qui ont permis d'améliorer le texte. Elle demeure seule responsable de toute erreur ou insuffisance qui subsisterait. L'évolution rapide $d u$ sujet oblige l'auteur à préciser que ce débat a été achevé le 2 mars 2007.

\section{RÉFÉRENCES OMC-PROPOSITIONS}

OMC. Propositions relatives à un système multilatéral de notification et d'enregistrement des indications géographiques pour les vins et spiritueux, http://www.wto.org

Proposition conjointe des États-Unis, du Canada. Document TN/IP/W/5, du 23 novembre 2002, document TN/IP/W/9 du 13 avril 2004.
Proposition révisée des Communautés européennes et leurs États-membres. Document TN/IP/W/10, 14/06/05 ; document IP/C/W/107/Rev.1, du 22 juin 2000.

Proposition de Hong Kong et de la Chine. Document TN/IP/W/8, du 23 avril 2003. 